\title{
Neurodevelopmental outcomes after neonatal cardiac surgery: Role of cortical isoelectric activity
}

\author{
Laurie Seltzer, DO, ${ }^{\mathrm{a}}$ Michael F. Swartz, PhD, ${ }^{\mathrm{b}}$ Jennifer Kwon, MD, ${ }^{\mathrm{a}}$ James Burchfiel, PhD, ${ }^{\mathrm{a}}$ \\ Jill M. Cholette, MD, ${ }^{\mathrm{c}}$ Hongyue Wang, PhD, ${ }^{\mathrm{d}}$ Dawn Sweeney, MD, ${ }^{\mathrm{b}, \mathrm{e}}$ Heather R. Adams, MD, \\ Cecilia Meagher, $\mathrm{MD},{ }^{\mathrm{c}}$ Ron Angona, $\mathrm{CCP},{ }^{\mathrm{b}}$ Ronnie Guillet, $\mathrm{MD}, \mathrm{PhD},{ }^{\mathrm{f}}$ and George M. Alfieris, $\mathrm{MD}^{\mathrm{b}}$
}

\section{ABSTRACT}

Objectives: Neonates with congenital heart disease are at risk for impaired neurodevelopment after cardiac surgery. We hypothesized that intraoperative EEG activity may provide insight into future neurodevelopmental outcomes.

Methods: Neonates requiring surgery had continuous intraoperative EEG and hemodynamic monitoring. The level of EEG suppression was classified as either: slow and continuous; moderate burst suppression; severe burst suppression; or isoelectric (no brain activity for $>3$ minutes). Follow-up neurodevelopmental outcomes were assessed using the Vineland Adaptive Behavior Scale II (VinelandII).

Results: Twenty-one neonates requiring cardiac surgery developed a slow and continuous EEG pattern after general anesthesia. Ten neonates (48\%) maintained continuous brain electrical activity with moderate burst suppression as the maximum level of EEG suppression. Eleven neonates (52\%) developed severe burst suppression that progressed into an isoelectric state during the deep hypothermic period required for circulatory arrest. However, the duration of this state was significantly longer than circulatory arrest times $(111.1 \pm 50$ vs $22.3 \pm 17$ minutes; $P<.001)$. At a mean follow-up at $5.6 \pm 1.0$ years, compared with neonates with continuous brain electrical activity, neonates who developed an isoelectric state had lower Vineland-II scores in communication. There was an inverse relationship between composite Vineland-II scores and duration of isoelectric activity $(\mathrm{R}=-0.75, P=.01)$. Of neonates who experienced an isoelectric state, durations of $>90$ minutes were associated with the lowest Vineland-II scores $(125.0 \pm 2.6$ vs $81.1 \pm 12.7 ; P<.01)$.

Conclusions: The duration of cortical isoelectric states seems related to neurodevelopmental outcomes. Strategies using continuous EEG monitoring to minimize isoelectric states may be useful during complex congenital heart surgery. (J Thorac Cardiovasc Surg 2016;151:1137-44)

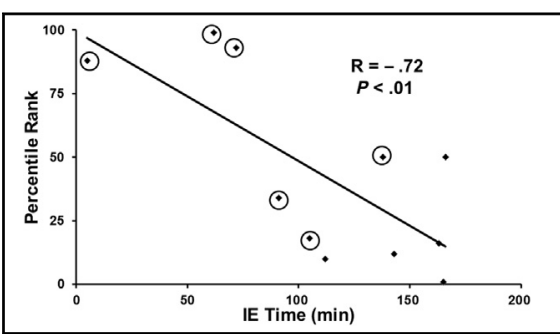

Relationship between the duration of isoelectric activity and neurodevelopmental outcomes.

Central Message

Longer duration of an intraoperative cerebral isoelectric state, as measured by EEG, in neonates undergoing surgery for congenital heart disease, is associated with worse neurodevelopmental outcomes.

\section{Perspective}

The use of intraoperative neurologic monitoring for neonates requiring pediatric cardiac surgery has been unreliable in detecting postoperative neurodevelopmental outcomes. This study found a correlation between worse such outcomes with longer duration of an isoelectric brain state, as measured by intraoperative EEG Thus, use of intraoperative EEG monitoring may help provide further insight into and ability to affect neurodevelopmental outcomes in this population.

See Editorial Commentary page 1145 .

\footnotetext{
From the Departments of ${ }^{\mathrm{a}}$ Neurology, ${ }^{\mathrm{b}}$ Surgery, ${ }^{\mathrm{c}}$ Pediatrics, ${ }^{\mathrm{d}}$ Statistics, ${ }^{\mathrm{e}}$ Anesthesia, and ${ }^{\mathrm{f}}$ Neonatology, University of Rochester Medical Center, Rochester, NY.

Funding for this work was provided $\mathrm{NIH}$, to the University of Rochester Medical Center, Department of Neurology (K-12NS 066098) (to L.S.).

Read at the 95th Annual Meeting of The American Association for Thoracic Surgery, Seattle, Washington, April 25-29, 2015.

L.S. and M.F.S. contributed equally to this work.

Received for publication April 20, 2015; revisions received Oct 6, 2015; accepted for publication Oct 11, 2015; available ahead of print Dec 18, 2015.

Address for reprints: Michael F. Swartz, PhD, University of Rochester Medical

Center, Box Surg/Cardiac, 601 Elmwood Ave, Rochester, NY 14642 (E-mail:

Michael_Swartz@urmc.rochester.edu).

$0022-5223 / \$ 36.00$

Copyright (c) 2016 by The American Association for Thoracic Surgery

http://dx.doi.org/10.1016/j.jtcvs.2015.10.065
}

Neurodevelopmental delay is commonly diagnosed at 1 to 10 years after neonatal cardiac surgery, ${ }^{1-4}$ and likely results from a combination of pre- and intraoperative factors. ${ }^{1-8}$ However, despite increased awareness, the exact mechanism(s) responsible for neurodevelopmental delay remain elusive. In addition, only minimal advances have been made to improve neurodevelopmental outcomes in neonates during complex cardiac procedures. ${ }^{7,8}$

Although initial results of using selective antegrade regional perfusion were promising, ${ }^{9}$ data are conflicting as to whether this approach improves outcomes, compared with deep hypothermic circulatory arrest (DHCA). ${ }^{10}$ 


\section{Abbreviations and Acronyms \\ CBEA = continuous brain electrical activity \\ $\mathrm{CPB}=$ cardiopulmonary bypass \\ DHCA $=$ deep hypothermic circulatory arrest \\ EEG = electroencephalogram \\ HLHS = hypoplastic left heart syndrome \\ Vineland-II = Vineland Adaptive Behavior Scale II}

Furthermore, monitoring devices designed to identify and predict poor neurodevelopmental outcomes have not led to clear delineation of those at highest risk. Near-infrared spectroscopy, cerebral oximetry, and electroencephalograms (EEGs) have, in selected case reports, demonstrated benefit; however, that improvement has not been observed within a larger population..$^{11-14}$

In recent intraoperative EEG monitoring, during the repair procedure for neonates who had congenital heart disease, we observed distinct EEG patterns that were primarily dependent on body temperature. ${ }^{15}$ We found that an isoelectric state in the brain, defined as absence of brain activity, began before, and extended beyond, the DHCA time. Therefore, the aim of the current study was to determine the impact of an intraoperative cortical brain isoelectric state on intermediate-term neurodevelopmental outcomes.

\section{METHODS}

Institutional review board approval and parental consent were obtained. Neonates aged $<30$ days who required cardiac surgery, and were previously enrolled in a prospective observational study, ${ }^{15}$ were assessed for neurodevelopmental outcomes. Exclusion criteria included preoperative seizure activity, known central nervous system injury, and multiple extracardiac congenital anomalies. A limited central nervous system assessment was performed preoperatively by the treatment team, who assessed for gross neurologic dysfunction; a preoperative EEG, without sedation of the patient, was used to assess for seizures. Routine care at our institution did not include brain imaging prior to surgery, unless the clinical status suggested the presence of central nervous system dysfunction.

\section{Electroencephalogram Recordings}

Gold-plated EEG electrodes were affixed to the scalp, and lead placement was in accordance with the international 10-20 system with the exception of FP1 (left frontal lobe) and FP2 (right frontal lobe), as described elsewhere. ${ }^{15}$ A baseline 30-minute preoperative EEG was obtained in all neonates 24 hours prior to surgery, with the patients awake and without sedation. The EEG recordings resumed upon arrival at the operating room and were made continuously for the duration of the procedure.

Analysis of EEG recordings was performed postprocedure by 2 of the investigators, under blind study conditions, and fluctuations in EEG activity were linked to events recorded from within the intraoperative record. The EEG activity was classified as either: (1) appropriate for age: normal for patient's age; (2) slow and continuous: EEG with slower frequencies but remaining continuous; (3) having moderate burst suppression: intervals of $<30$ seconds between bursts; (4) having severe burst suppression: intervals of 31 to 179 seconds between bursts; or (5) isoelectric: no brain activity for $>180$ seconds (Figure 1 ). ${ }^{15}$ Intraoperative events were extracted from the intraoperative record according to a predetermined protocol, by a single individual, under conditions of study blinding to patients' EEG data.

\section{Operative Technique}

All neonates required cardiopulmonary bypass (CPB) for either cardiac repair or palliation. Fentanyl and pancuronium were used for anesthetic induction, and anesthesia was maintained with fentanyl and isoflurane throughout the remainder of the procedure. Arterial cannulation was aortic in all cases, except for neonates who received regional perfusion.

Regional perfusion $(n=4)$ was used in patients with hypoplastic left heart syndrome (HLHS) and was accomplished after the cannulation of a 3.5-mm graft sewn to the innominate artery. Next, neonates were cooled to 18 to $20^{\circ} \mathrm{C}$, with flows of 20 to $30 \mathrm{ml} / \mathrm{kg}$. In the absence of DHCA, all patients were cooled to 32 to $34^{\circ} \mathrm{C}$. Cooling was achieved by lowering the blood temperature by $1^{\circ} \mathrm{C}$ every 1 to 2 minutes. Rewarming was initiated, typically after removal of the aortic crossclamp, and achieved via increasing the arterial blood temperature by $1^{\circ} \mathrm{C}$ every 3 minutes. While patients were on $\mathrm{CPB}$, blood glucose was maintained between 80 and $200 \mathrm{~g} / \mathrm{dL}$ (using insulin and 50\% dextrose solution [D50]); $\mathrm{PaO}_{2}$ (ratio of partial pressure arterial oxygen and fraction of inspired oxygen) was maintained at $>100 \mathrm{~mm} \mathrm{Hg} ; \mathrm{PaCO}_{2}$ (partial pressure of carbon dioxide in the arterial blood) levels at 35 to $45 \mathrm{~mm} \mathrm{Hg}$; and hemoglobin $>7.0 \mathrm{~g} / \mathrm{dL}$, corrected to $37^{\circ} \mathrm{C}$, utilizing $\mathrm{pH}$-stat cooling and alpha-stat rewarming.

\section{Follow-up}

In 2013, the parents of the 32 neonates who previously had intraoperative EEG recordings ${ }^{15}$ were contacted and asked to participate in the assessment of their child's neurodevelopmental outcome. The Vineland Adaptive Behavior Scale, second edition (Vineland-II) was used to evaluate attainment of neurodevelopmental milestones. The Vineland-II is a reliable, well-validated omnibus measure of adaptive function that evaluates adaptive skills in multiple domains, including communication, motor function, activities of daily living, social skills, and maladaptive behaviors. ${ }^{16,17}$ The Vineland-II, which is appropriate for use across the lifespan, was chosen because of the age of the participants, and because it allows for evaluation of multiple areas of development. ${ }^{16,17}$

Parents who agreed to participate in this aspect of the study were mailed the Vineland-II questionnaire and asked to complete and return it. A postcompletion telephone interview was performed by 1 of the investigators, under blind conditions, who was trained in the administration of the Vineland-II and could thereby ensure the accuracy and completeness of parental responses. The questionnaires were scored using standard software ${ }^{18}$ that generated an age-adjusted score-reflecting global performance in relation to same-age peers-and an individual score for each functional domain (communication, activities of daily living, social skills, and motor skills). In addition, $z$-values for the composite Vineland-II score (summarizing global adaptive skills across all areas assessed), as well as from individual domains, were determined to demonstrate the degree of neurodevelopment compared with the mean for age $(z$-value mean $=0 ; \mathrm{SD}=1.0)$.

\section{Statistical Analysis}

Descriptive statistics were used to describe the study measures, such as mean, SD, median with 95th percentiles, frequency, and percentage. Continuous variables were compared between study groups, using a Mann-Whitney $U$ test; categoric variables were compared using Pearson $\chi^{2}$ analysis or the Fisher exact test, where appropriate. The Pearson correlation coefficient was calculated to evaluate the associations of the duration of isoelectric activity, and of the DHCA, with the Vineland-II composite score. Multiple linear regression analysis was performed to test the effects of hypothermia, diagnosis, and duration of an isoelectric state on composite Vineland-II scores.

The $F$-test and standard diagnostic measures, such as residual plots, were used to check model adequacy and goodness-of-fit. Multicollinearity between predictor variables was assessed using multiple correlations. All statistics were completed using SPSS, version 21 (SPSS, Inc, Chicago, 

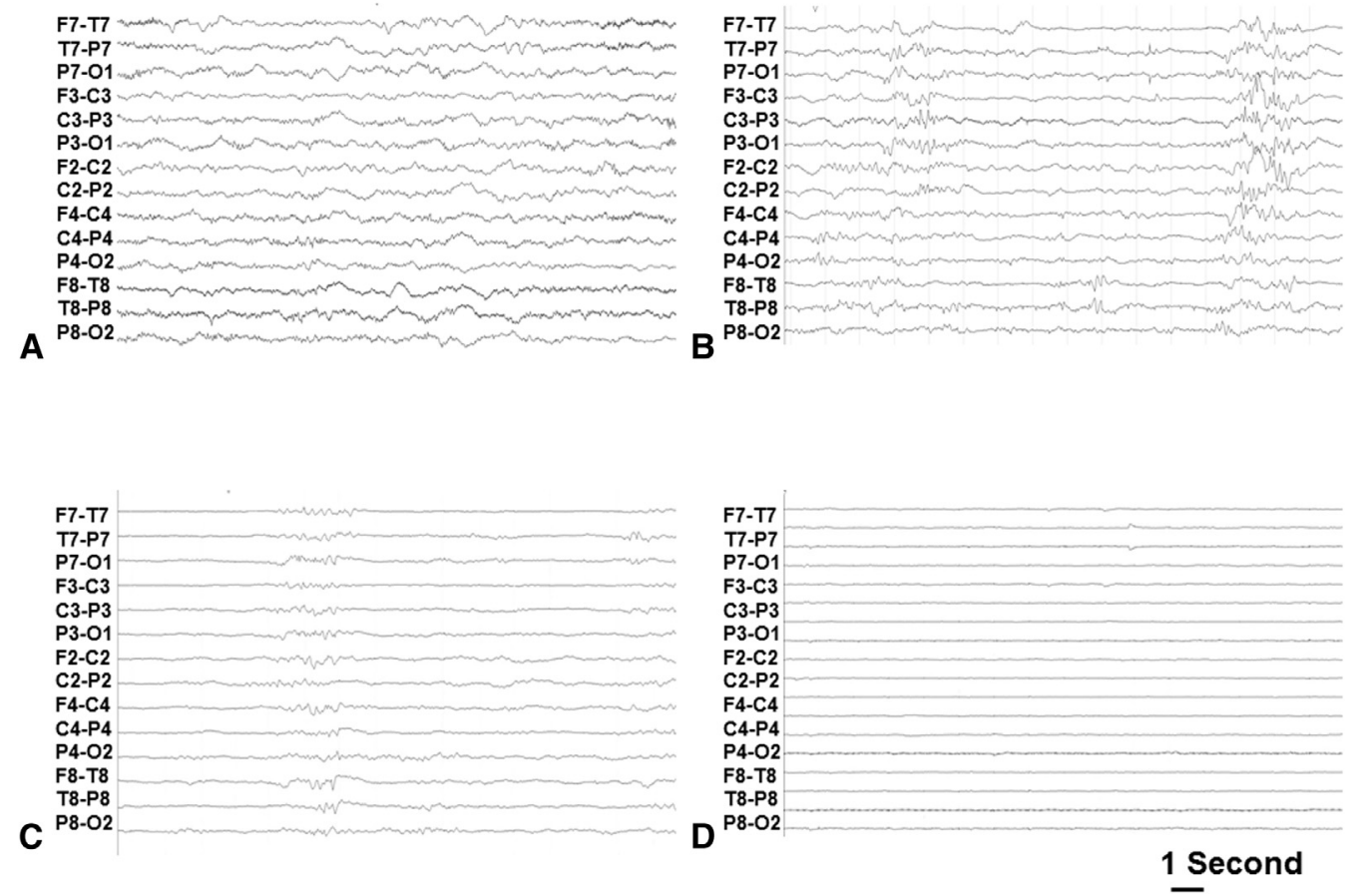

FIGURE 1. Representative EEGs demonstrating: (A) slow and continuous; (B) moderate burst suppression; (C) severe burst suppression; and (D) isoelectric state. $F$, Frontal; $T$, temporal; $P$, parietal; $O$, occipital; $C$, cortex.

Ill) or GraphPad Prism (GraphPad Software, Inc). The Bonferroni correction was applied, when appropriate, to ensure that the type I error rate remained $\leq 5 \%$. A power calculation was not performed, because this study was a follow-up of previously enrolled patients.

\section{RESULTS}

Of 32 neonates, 6 died during the follow-up period, and 2 were otherwise lost to follow-up. For 3 subjects, the parents did not complete the survey correctly, and appropriate values could not be determined from a phone interview. Therefore, these 3 children were excluded. The EEG data and subsequent neurodevelopmental outcomes were analyzed from the 21 remaining patients (Table 1). Baseline demographics demonstrated various congenital heart diseases, in which only 1 neonate with tetralogy of Fallot was cyanotic.

\section{Intraoperative Electroencephalogram Patterns}

Baseline EEG measurements were normal for gestational age in 19 neonates, and suggested relative dysmaturity in 2 neonates (consistent with a gestational age of 2 weeks younger). With the induction of general anesthesia, the EEG changed, from normal for gestational age to slow and continuous. Moderate burst suppression was typically observed at the initiation of CPB for 15 of $21(71.4 \%)$ neonates, and was present in all patients while they were on CPB. Ten neonates $(48 \%)$ maintained continuous brain electrical activity (CBEA), and the maximum level of EEG burst suppression throughout the procedure remained moderate. All neonates who cooled to $<32^{\circ} \mathrm{C}$ developed severe burst suppression, and a subsequent isoelectric state (Figure 1).

The mean arterial pressure, flow during CPB, and temperature at the initiation of each EEG class, are shown in Figure 2. Although at each stage, fluctuations were seen in mean arterial pressure and flow, these variables remained fairly constant despite changes in EEG suppression. In contrast, a decrease in temperature was the only variable directly associated with EEG suppression. Similarly, an increase in temperature at the conclusion of the procedure was associated with decreased EEG suppression. The onset of an isoelectric state occurred prior to DHCA in most of the neonates (10 of 11 ), at $23.2 \pm 3.8^{\circ} \mathrm{C}$.

Further, the return of EEG activity from an isoelectric state to severe burst suppression occurred at $34.0 \pm 2.4^{\circ} \mathrm{C}$, nearly $4^{\circ} \mathrm{C}$ warmer than at severe burst suppression initiation. As a consequence, isoelectric times were significantly longer than DHCA times $(111.1 \pm 50$ vs $22 \pm 17$ minutes, respectively; $P<.01)$. No differences were found in mean arterial pressure or flow on CPB, for neonates who maintained CBEA, versus those who developed an isoelectric state.

\section{Neurodevelopmental Outcomes}

Follow-up was obtained at age $5.6 \pm 1.0$ years. Six children had interim events, all of which were reoperation and/or catheter-based reintervention: 5 as subsequent 
TABLE 1. Patient- and surgery-related variables

\begin{tabular}{|c|c|c|c|}
\hline Variable & IE $(\mathbf{n}=11)$ & $\operatorname{CBEA}(n=10)$ & $\begin{array}{c}P \\
\text { value }\end{array}$ \\
\hline \multicolumn{4}{|l|}{ Preoperative demographics } \\
\hline Age at operation (d) & $7.0 \pm 2.3$ & $7.7 \pm 2.5$ & .6 \\
\hline Gestational age (mo) & $39.2 \pm 0.6$ & $38.9 \pm 1.2$ & .5 \\
\hline Gender, female & $7(64)$ & $1(10)$ & $.02 *$ \\
\hline Weight (kg) & $3.1 \pm 0.5$ & $3.4 \pm 0.4$ & .2 \\
\hline Head circumference $(\mathrm{cm})$ & $35.5 \pm 5.3$ & $34.6 \pm 1.3$ & .7 \\
\hline Prenatal diagnosis & $4(36)$ & $3(30)$ & .9 \\
\hline \multicolumn{4}{|l|}{ Cardiac diagnosis } \\
\hline HLHS or variant & 5 & 0 & $.03 *$ \\
\hline D-TGA & 1 & 9 & $.003^{*}$ \\
\hline Tetralogy of Fallot & 0 & 1 & 1.0 \\
\hline $\begin{array}{l}\text { CoA/arch hypoplasia } \\
\text { >BiV RPR }\end{array}$ & 5 & 0 & $.03 *$ \\
\hline \multicolumn{4}{|l|}{ Operative } \\
\hline CPB time (min) & $146.0 \pm 33.3$ & $173.9 \pm 23.7$ & $.03 *$ \\
\hline Crossclamp time (min) & $74.8 \pm 25.2$ & $120.9 \pm 15.8$ & $<.01 *$ \\
\hline DHCA time (min) & $22.3 \pm 17.0$ & 0 & \\
\hline Regional perfusion & $4(36)$ & 0 & \\
\hline $\begin{array}{l}\text { Regional perfusion time } \\
\text { (min) }\end{array}$ & $69.5 \pm 13.4$ & 0 & \\
\hline $\begin{array}{l}\text { Lowest temperature } \\
\text { on } \mathrm{CPB}\left({ }^{\circ} \mathrm{C}\right)\end{array}$ & $20.7 \pm 2.4$ & $33.7 \pm 0.8$ & $<.01 *$ \\
\hline \multicolumn{4}{|l|}{ Postoperative } \\
\hline Peak lactate (mg/dL) & $7.5 \pm 2.7$ & $6.9 \pm 3.0$ & .4 \\
\hline Ventilation duration (d) & $3.8 \pm 1.2$ & $3.0 \pm 1.4$ & .1 \\
\hline $\begin{array}{l}\text { Length of stay }(\mathrm{d}), \\
\text { median (range) }\end{array}$ & $14(10.8,22.2)$ & $9.5(6.7,16.1)$ & .07 \\
\hline \multicolumn{4}{|c|}{ Follow-up (number of interim events) } \\
\hline 0 & $6(54.5)$ & $9(90)$ & \\
\hline 1 & $1(9.1)$ & $1(10)$ & \\
\hline$\geq 2$ & $4(36.4)$ & 0 & \\
\hline
\end{tabular}

Values are $\mathrm{n} ; \mathrm{n}(\%)$; or mean $\pm \mathrm{SD}$, unless otherwise indicated. $I E$, Isoelectricity; $C B E A$, continuous brain electrical activity; $H L H S$, hypoplastic left heart syndrome; $D-T G A$, transposition of the great arteries; $C O A$, coarctation; $B i V$, biventricular; $R P R$, repair; $C P B$, cardiopulmonary bypass; $D H C A$, deep hypothermic circulatory arrest. $* P<.05$.

staging for HLHS; 1 for augmentation of a transannular patch. As demonstrated in Table 2, children who were cooled, and subsequently developed an isoelectric state, had significantly lower Vineland-II scores in communication, which evaluates receptive comprehension, oral expressive language, and written language skills. The 3 children with the highest composite Vineland-II and communication scores had the shortest durations of an isoelectric state; neonates with times $>90$ minutes had the lowest Vineland-II scores $(81.1 \pm 12.7$ vs $125.0 \pm 2.6$, respectively; $P<.01$ ).

To test the association between neurodevelopmental outcome and DHCA or an isoelectric state, linear regression was performed, and demonstrated an inverse correlation between duration of isoelectric activity and the composite Vineland-II score (Figure 3). However, within the limits of this dataset, no correlation was observed between
DHCA times and the composite Vineland-II scores $(R=0.2 ; P=.6)$. Multiple regression analysis was performed to further evaluate the impact of the duration of the isoelectric state with hypothermia, and of cardiac diagnosis on the Vineland-II composite score. Multiple regression analysis demonstrated that only the duration of the isoelectric state was associated with lower Vineland-II composite scores $(P=.007 ; 95 \%$ confidence interval: 0.55 to 0.18$)$; whereas temperature $(P=.22 ; 95 \%$ confidence interval: -3.3 to 0.8 ), and preoperative diagnosis $(P=.6-.4 ; 95 \%$ confidence interval: -24.6 to 57.4), were not significantly associated with lower Vineland-II composite scores.

\section{DISCUSSION}

In this series, neonates who required DHCA, and developed isoelectric EEGs during surgery, had lower Vineland-II scores in communication at $5.6 \pm 1.0$ years of follow-up. Neonates with isoelectric-state times $>90$ minutes had the poorest neurodevelopmental outcomes. Further, the duration of the isoelectric period on EEG, and not the duration of DHCA, inversely and independently correlated with a lower composite Vineland-II score.

The observed intraoperative EEG patterns have been reported for $>30$ years, but without demonstrating any clinical correlation. ${ }^{14}$ Previously, investigators have assessed the impact of EEG monitoring on predicting neurodevelopmental outcomes after congenital heart surgery. ${ }^{11}$ However, examination of the neurologic function was limited to the postoperative period and was not at an intermediate-term follow-up, the point at which others have previously observed the greatest neurodevelopmental impact. ${ }^{4,19}$

An isoelectric state is thought to be neuroprotective by decreasing the cerebral metabolism, thereby creating conditions that may be optimal during periods of hypoperfusion. ${ }^{20}$ However, the extent of the isoelectric state within these neonates was deep, profound, and continuous; in some cases, it approached 3 hours in duration. Further, to our knowledge, no benefit comes from lowering the body temperature below the point of isoelectricity, which in most cases developed at approximately $25^{\circ} \mathrm{C}$. However, the question is unresolved as to whether minimization of hypothermia, and the subsequent duration of an isoelectric state, will result in improved neurodevelopmental outcomes, or exacerbate brain hypoxia at the time of the operation, potentially resulting in worse neurologic function.

Monitoring of EEG is only 1 type of intraoperative neurologic monitoring that can be performed during the repair/palliation of congenital heart disease. Unlike transcranial Doppler and cerebral oxygenation, EEG monitoring provides information about the overall cortical function of the patient. ${ }^{11,14}$ Transcranial Doppler and cerebral 

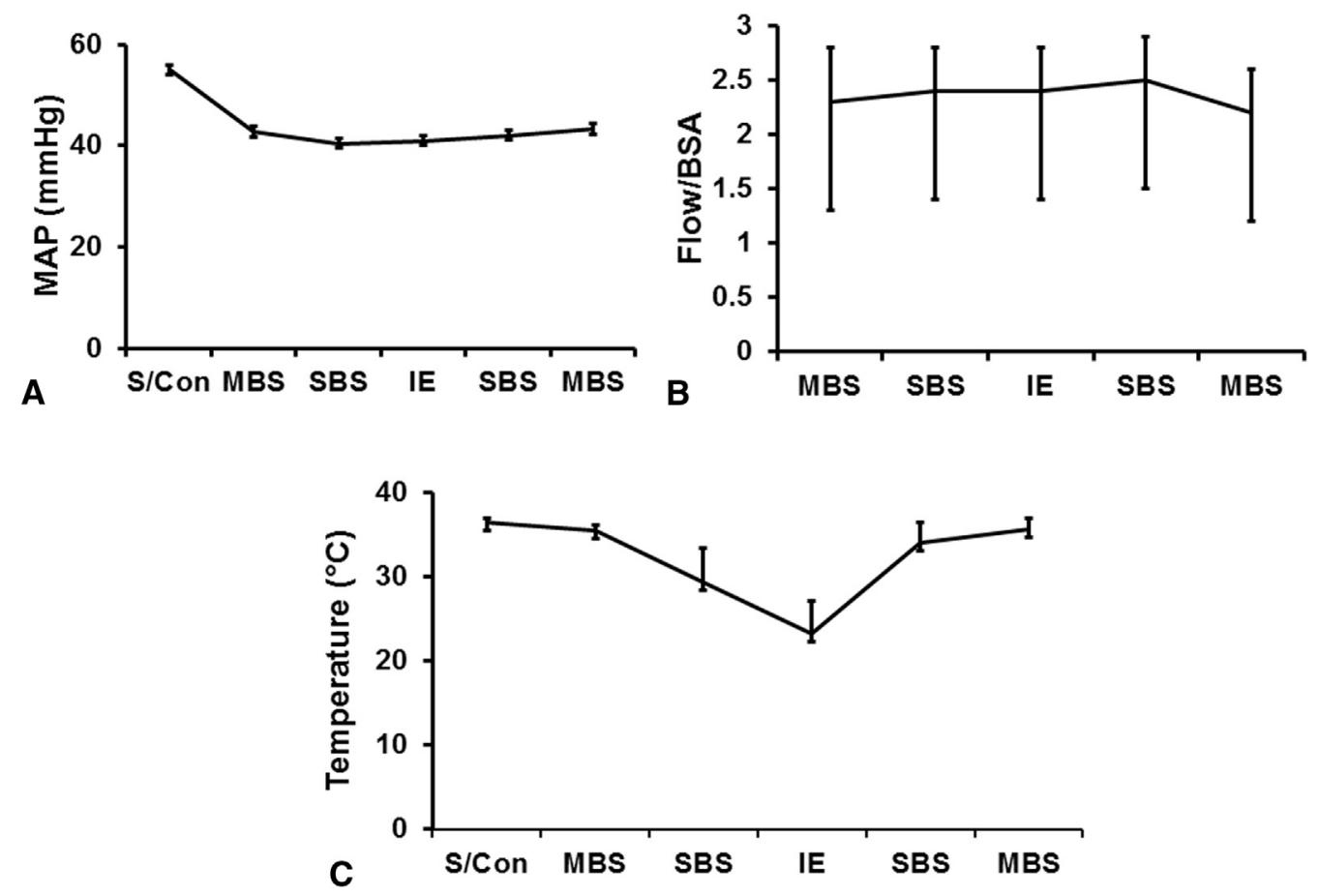

FIGURE 2. A, Mean arterial blood pressure; (B) flow during cardiopulmonary bypass; and (C) temperature at the initiation of each stage of electroencephalogram suppression during the repair of congenital heart disease. MAP, Mean arterial pressure; $S / C o n$, slow and continuous; $M B S$, moderate burst suppression; $S B S$, severe burst suppression; $I E$, isoelectric activity; $B S A$, body surface area.

oximetry provide information only about overall blood flow to various regions of the brain and do not reflect the hypothermia-induced injury, which is likely substantial.

Previous reports have demonstrated that variables including younger gestational age, lower birth weight, and longer hospital length of stay are associated with worse neurodevelopmental outcomes. ${ }^{1-3}$ Unfortunately, due to the limited number of subjects, the current study is underpowered for identifying any association of an isoelectric state, these variables, and overall neurodevelopmental outcomes. The question remains of whether the duration of DHCA is an independent risk factor for poor neurodevelopmental outcomes. ${ }^{21-23}$

Early outcomes demonstrated no difference from the single-ventricle reconstruction trial, ${ }^{4}$ whereas intermediateterm data suggested that the duration of DHCA was associated with poor neurodevelopmental outcomes. ${ }^{22,23}$
The Boston Circulatory Arrest Study found that DCHA times $>40$ minutes were associated with poor neurodevelopmental outcomes at 8 years, but not at 16 years. $^{23}$ Most of our subjects had DHCA times of $<40$ minutes, which may explain why DHCA was not inversely correlated with the composite Vineland-II score.

\section{Limitations}

This work has several limitations. Subjects did not undergo preoperative imaging; therefore, the existence of preoperative central nervous system pathology cannot be excluded and could have confounded subsequent results. Monitoring of EEG was not coupled with other technology, such as near-infrared spectroscopy or cerebral oximetry, which would have provided additional intraoperative data. The small sample size and cohort represent a diverse group of patients who have varying cardiac anatomy and diagnosis,

TABLE 2. Standard scores and $z$-scores from the Vineland-II

\begin{tabular}{lccccc}
\hline & \multicolumn{2}{c}{ IE $(\mathbf{n}=\mathbf{1 1})$} & & \multicolumn{2}{c}{ CBEA $(\mathbf{n}=\mathbf{1 0})$} \\
\cline { 2 - 3 } \multicolumn{1}{c}{ Vineland-II measure } & Standard & $z$ & & Standard & \multicolumn{2}{c}{$\boldsymbol{z}$} \\
\hline Communication standard & $98.2 \pm 20.2$ & $-0.3 \pm 1.3$ & & $108.8 \pm 9.8$ & $0.6 \pm 0.6$ \\
Daily living & $90.3 \pm 22.5$ & $-0.6 \pm 1.5$ & & $97.6 \pm 8.6$ & $-0.2 \pm 0.6$ \\
Socialization & $96.5 \pm 22.4$ & $-0.2 \pm 1.5$ & & $99.7 \pm 14.4$ & $0.0 \pm 0.9$ \\
Motor skills & $89.6 \pm 23.0$ & $-0.7 \pm 1.5$ & & $96.5 \pm 10.3$ & $-0.3 \pm 0.7$ \\
Composite Vineland-II score & $93.1 \pm 23.1$ & $-0.5 \pm 1.5$ & & $100.4 \pm 9.6$ & .7 \\
Total sum Vineland-II score & $351.1 \pm 100.0$ & & $392.0 \pm 49.0$ & .4 \\
\hline
\end{tabular}

Values are mean $\pm \mathrm{SD}$, unless otherwise indicated. Vineland-II score, Vineland Adaptive Behavior Scale II; $I E$, Isoelectricity; $C B E A$, continuous brain electrical activity. *P<.05. 


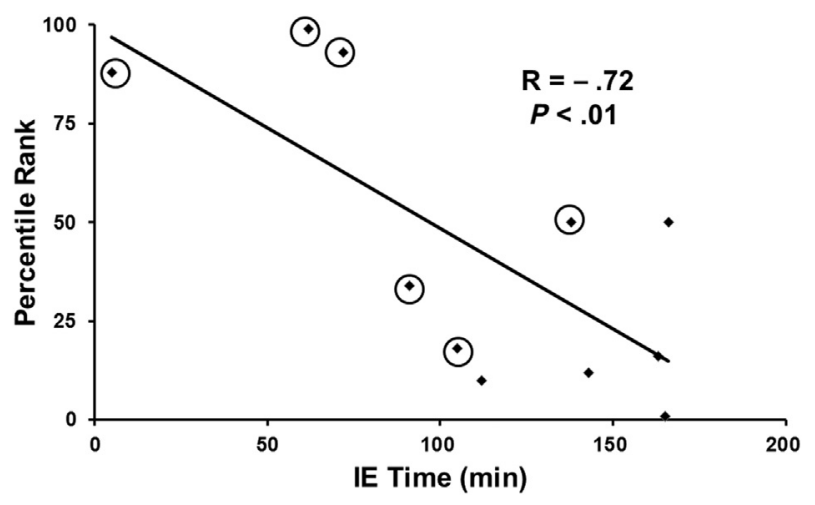

FIGURE 3. Linear regression between the composite Vineland Adaptive Behavior Scale-II score and the duration of an isoelectric state. Circled points represent neonates who received a biventricular cardiac repair. $I E$, Isoelectricity.

type of surgical intervention and subsequent operative details, and reintervention and neurodevelopmental outcomes.

These variables confound this study, and the possibility of differentiating among these differences is limited. Therefore, the correlations observed between the duration of the isoelectric state and the neurodevelopmental outcomes may be the result of these differences. Last, neurodevelopmental outcomes were assessed by survey only.

\section{CONCLUSIONS}

Results of this study demonstrate for the first time an inverse correlation between the duration of an isoelectric state in the brain, initiated primarily by profound hypothermia, and the development of poor neurodevelopmental outcomes. Neonates requiring surgery that resulted in an isoelectric-state duration of $>90$ minutes had the poorest outcomes. Future studies will investigate the use of moderate hypothermia and real-time EEG monitoring as a way to potentially moderate EEG suppression, which may lead to improved neurodevelopmental outcomes.

\section{Conflict of Interest Statement}

Authors have nothing to disclose with regard to commercial support.

You can watch a Webcast of this AATS meeting presentation by going to: http://webcast.aats.org/2015/Video/ Tuesday/04-28-15_6A_1655_Swartz.mp4.

The authors thank Joan Merzbach for her clinical expertise in assisting with this project.

\section{References}

1. Fuller S, Rajagopalan R, Jarvik GP, Gerdes M, Bernbaum J, Wernovsky G, et al. J. Maxwell Chamberlin Memorial Paper for congenital heart surgery. Deep hypothermic circulatory arrest does not impair neurodevelopmental outcome in school-age children after infant cardiac surgery. Ann Thorac Surg. 2010;90: 1985-94.
2. Gaynor JW, Wernovsky G, Jarvik GP, Bernbaum J, Gerdes M, Zackai E, et al. Patient characteristics are important determinants of neurodevelopmental outcomes at one year of age after neonatal and infant cardiac surgery. J Thorac Cardiovasc Surg. 2007;133:1344-53.

3. Newburger JW, Sleeper LA, Bellinger DC, Goldberg CS, Tabbutt S, Lu M, et al. Early developmental outcome in children with hypoplastic left heart syndrome and related anomalies: the single ventricle reconstruction trial. Circulation. 2012;125:2081-91.

4. Hövels-Gürich HH, Seghaye MC, Schnitker R, Wiesner M, Huber W, Minkenberg R, et al. Long-term neurodevelopmental outcomes in school-aged children after neonatal arterial switch operation. J Thorac Cardiovasc Surg. 2002; $124: 448-58$.

5. Williams GD, Ramamoorthy C. Brain monitoring and protection during pediatric cardiac surgery. Semin Cardiothorac Vasc Anesth. 2007;11:23-33.

6. Dimitropoulos A, McQuillen PS, Sethi V, Moosa A, Chau V, Xu D, et al. Brain injury and development in newborns with critical congenital heart disease. Neurology. 2013;81:241-8.

7. Martinez-Biarge M, Jowet VC, Cowan FM, Wusthoff CJ. Neurodevelopmental outcome in children with congenital heart disease. Semin Fetal Neonatal Med. 2013;18:279-85.

8. DiNardo JA. Should what we know about neurobehavioral development, complex congenital heart disease, and brain maturation affect the timing of corrective cardiac surgery? Paediatr Anaesth. 2011;21:781-6.

9. Pigula FA, Nemoto EM, Griffith BP, Siewers RD. Regional low-flow perfusion provides cerebral circulatory support during neonatal aortic arch reconstruction. J Thorac Cardiovasc Surg. 2000;119:331-9.

10. Goldberg CS, Bove EL, Devaney EJ, Mollen E, Schwartz E, Tindall S, et al. A randomized clinical trial of regional cerebral perfusion versus deep hypothermic circulatory arrest: outcomes for infants with functional single ventricle. J Thorac Cardiovasc Surg. 2007;133:880-7.

11. Miller G, Rodichok LD, Baylen BG, Myers JL. EEG changes during open heart surgery on infants aged 6 months or less: relationship to early neurologic morbidity. Pediatr Neurol. 1994;10:124-30.

12. Hirsch JC, Charpie JR, Ohye RG, Gurnery JG. Near Infrared spectroscopy (NIRS) should not be standard of care for postoperative management. Semin Thorac Cardiovasc Pediatr Cardiol Surg Ann. 2010;13:51-4.

13. Sanchez-de-Toledo J, Chrysostomou C, Munoz R, Lichenstein B, SaoAvilès CA, Wearden PD, et al. Cerebral regional oxygen saturation and serum neuromarkers for the prediction of adverse neurologic outcome in pediatric cardiac surgery. Neurocrit Care. 2014;21:133-9.

14. Reilly EL, Brunberg JA, Doty DB. The effect of deep hypothermia and total circulatory arrest on the electroencephalogram in children. Electroencephalogr Clin Neurophysiol. 1974;36:661-7.

15. Seltzer LE, Swartz M, Kwon JM, Burchfiel J, Alfieris GM, Guillet R. Intraoperative EEG predicts postoperative seizures in infants with congenital heart disease. Ped Neurol. 2014;50:313-7.

16. Wynn J, Aspelund G, Zygmunt A, Stolar CJ, Mychaliska G, Butcher J, et al. Developmental outcomes of children with congenital diaphragmatic hernia: a multicenter prospective study. J Ped Surg. 2013;45:1995-2004.

17. Gleason K, Coster W. An ICF-CY-based content analysis of the Vineland Adaptive Behavior Scales-II. J Intellect Dev Disabil. 2012;37:285-93.

18. Sparrow SS, Cicchetti DV, Balla DA. Vineland Adaptive Behavior Scales, second edition. 2012;1:1-6.

19. Mulkey SB, Swearingen CJ, Melguizo MS, Reeves RN, Rowel JA, Gibson N, et al. Academic proficiency in children after early congenital heart disease surgery. Pediatr Cardiol. 2014;35:344-52.

20. Nakashima K, Todd MM. Effects of hypothermia on the rate of excitatory amino acid release after ischemic depolarization. Stroke. 1996;27:913-8.

21. Majnemer A, Limperopoulos C, Shevell M, Rohlicek C, Rosenblatt B, Techervenkov C. Developmental and functional outcomes at school entry in children with congenital heart defects. J Pediatr. 2008;153:55-60.

22. Forbess JM, Visconti KJ, Hancock-Friesen C, Howe RC, Bellinger DC, Jonas RA. Neurodevelopmental outcome after congenital heart surgery: results from an institutional registry. Circulation. 2002;106(12 Suppl 1):I95-102.

23. Bellinger DC, Wypij D, Rivkin MJ, Demaso DR, Robertson RL Jr, DunbarMasterson C. Adolescents with d-transposition of the great arteries corrected with the arterial switch procedure: neuropsychological assessment and structural brain imaging. Circulation. 2011;124:1361-9.

Key Words: neurodevelopmental outcomes, deep hypothermic circulatory arrest, EEG 


\section{Discussion}

Dr J. Gaynor (Philadelphia, Pa). Dr Swartz, I would like to congratulate you on a very nice and important study. Thank you for allowing me to review the manuscript.

As survival has improved after surgery, for even complex congenital heart disease, neurodevelopmental dysfunction is increasingly recognized as a common adverse outcome that has important adverse effects on quality of life, educational attainments, and employment opportunities for our patients. As this has become more evident, it is important to develop methods to let us understand the underlying mechanisms of brain injury and develop ways to monitor this and hopefully treat and prevent brain injury. I think this study looking at the role of intraoperative EEG is potentially very important.

There are basically 2 major concerns I have on reviewing the study. First, I had several of our neurologists review the study, and I have always been taught that with hypothermia, the development of isoelectric activity signifies suppression of cerebral metabolic activity, which is the goal of hypothermia, to provide a protected time. Therefore, it is counterintuitive to me that the period of isoelectric activity is associated with a worse outcome, because it seems that you are achieving your goal of suppressing metabolism. We need additional studies, obviously, to begin to look at the relationship of the isoelectric activity to cerebral metabolism, but I find that result to be very counterintuitive, and I would like you to speculate on why that period is associated with worse outcomes.

Second, and you alluded to this in your results, is that the management strategies, particularly cooling and circulatory arrest, are highly tied to the type of heart disease. You cannot separate the cooling from the circulatory arrest, particularly from HLHS. We know that children with HLHS have many other factors that are not possible to control for. Because of the small sample size, you were not able to do a multivariable analysis. Children with HLHS have very prolonged hospital stays, which can correlate with worse outcomes. They are hypoxic for often 2 to 3 years and undergo multiple operations.

In contrast, children with DTGA have early complete repair to a biventricular circulation. They do not have prolonged hypoxemia. They usually have relatively short hospital stays. They do not require reoperation.

So I would argue that what you are seeing is not an impact of the isoelectric activity, but just the fact that it is linked to a type of heart disease that has many other causes. If you had a larger sample size, and were able to do a multivariable analysis, I would imagine that this would fall out. I think that is particularly likely given that I do not really understand - and I hope you can provide one-a mechanism by which that period of isoelectric activity would cause worse outcomes.
I enjoyed the paper very much.

Dr Swartz. Thank you, Dr Gaynor. Those are very insightful comments. We agree that EEG suppression is associated with a decrease in cerebral metabolism to the point of isoelectricity. However, no metabolic advantage comes from continually lowering the temperature below $25^{\circ} \mathrm{C}$ past the point of isoelectricity. We believe that lowering the body temperature to $<25^{\circ} \mathrm{C}$ may be detrimental.

In addition, the EEG suppression that we observed was profound, and extended for a vast period of time-in some patients, up to 3 hours. We believe that, potentially, this duration of overall suppression in conjunction with CPB may be even more detrimental. Last, the cold shock proteins are thought to be part of the molecular mechanisms associated with the protective effects of hypothermia. They are not upregulated at $18^{\circ} \mathrm{C}$. In summary, what we would speculate is that EEG suppression beyond isoelectric activity below $25^{\circ} \mathrm{C}$ may be more detrimental than it is just at $25^{\circ} \mathrm{C}$, meaning that too much of a good thing may be bad.

Dr Gaynor. I think that is a possibility, but very few data are available on moderate hypothermia and neurodevelopmental outcomes. In a recent study from Melbourne, in which they used regional cerebral perfusion and moderate hypothermia, they found that one third of their patients seized throughout the operation. So I think we have to be very careful about making the assumption that moderate hypothermia is going to provide the same degree of protection. So I think it is very interesting. Again, I think it is so linked to the type of cardiac disease in this study. There are so many other factors. Unless you control for length of stay, multiple different operations, and prolonged hypoxemia, it is going to be very, very difficult to sort it out.

Dr Swartz. Certainly we agree that this is a diverse and heterogeneous population, particularly within the isoelectric group, made up of 6 children with biventricular repairs, and 5 children with single-ventricle palliations. However, if you look at the linear regression, both the biventricular children and the single-ventricle children both fall along the line. We agree that, unfortunately, we were not able to do a multivariate analysis, and we are aiming to further study this area in hopes of validating these results.

Dr Gaynor. But it is also important that your $\mathrm{R}$ value is only about 0.07 for the linear relationship. I remember that figure.

Dr Swartz. The R value is 0.7 .

Dr Gaynor. Was it 0.7 ? Okay, excuse me, then that is my mistake. But I think it is, again, a small group, which may be a factor. But unless you can sort out all the other factors, I think I would be very careful about counseling people to do the circulatory arrest without deep hypothermia.

Dr Swartz. Thank you.

Dr E. Dean McKenzie (Houston, Tex). I want to congratulate you on a great presentation. Are you using 
intraoperative EEG monitoring as a research protocol, or is this part of your standard of care in your unit? Are you, or your anesthesiologist or perfusionist, using it as a tool to manage CPB?

Dr Swartz. We have recently moved to making the intraoperative EEG monitoring the standard of care. It is not currently a research protocol. We are specifically looking at both the intraoperative and the postoperative EEGs for seizure activity. However, the EEG data are not obtained in real time, meaning the EEGs are read 24 hours after they are collected.

Dr Carl L. Backer (Chicago, Ill). Are you using the EEG monitoring in the postoperative period? Are you keeping those scalp electrodes on and looking at the EEG in the intensive care unit after surgery?

Dr Swartz. For this cohort, we kept the EEG monitoring continuous for 48 hours postoperatively.

Dr Backer. What percentage of those patients had seizures in the postoperative period?

Dr Swartz. From the original cohort, 3 of 32 neonates developed seizures. Two of those neonates died in the interval period, resulting in 1 neonate who had a seizure from the isoelectric group.

Dr Backer. So this goes along with some data that are coming out in a paper from Dr Gaynor's institution (Children's Hospital of Philadelphia). The American Clinical Neurophysiology Society (ACNS) is recommending continuous postoperative EEG monitoring in all neonates undergoing neonatal cardiac surgery. The Children's Hospital of Philadelphia study showed that of neonates who had seizures, $85 \%$ were detected only by EEG monitoring. Within the seizure group, there was a very high mortality, just like you reported. My question for the entire audience is: Could I see a show of hands of how many people are routinely monitoring EEGs, first, in the operating room.

(Show of hands) So, I see 2 or 3 hands out of a huge audience. How about in the postoperative period? Are programs monitoring the EEG in the postoperative period, for neonates having cardiac surgery?

(Show of hands) Now I see only 3 or 4 hands of at least several hundred people in this auditorium. So, now there is this guideline from the ACNS, but we as a society are not following the recommendation for various reasons. We are left with an issue that we are going to have to resolve in some fashion.

Dr Can Yerebakan (Giessen, Germany). Very nice presentation. Thank you for the data. One more question: Do you use another method for neurological monitoring during the operation, or postoperatively, such as near-infrared spectroscopy? If yes, do you see any correlation with your data?

Dr Swartz. We did not during the study period. We have started using cerebral oximetry in all patients who require deep hypothermic circulatory arrest, but I cannot comment at this time on whether there is any association between them. 